\title{
Os teoremas de Stewart e de Heron e a demonstração nas aulas de matemática
}

\author{
Rudimar Luiz Nós Olga Harumi Saito \\ Departamento Acadêmico de Matemática, UTFPR \\ 80230-901, Curitiba, PR \\ E-mail: rudimarnos@gmail.com, ohsaito@gmail.com \\ Carlos Alberto Maziozeki de Oliveira* \\ CPM-PR - Colégio da Polícia Militar do Paraná \\ E-mail: ccoruja@hotmail.com
}

\begin{abstract}
Resumo: Apresenta-se neste trabalho o Teorema de Stewart e sua demonstração e emprega-se este teorema para demonstrar outros teoremas, assim como para solucionar problemas aplicados. Objetiva-se dessa maneira enfatizar a importância da demonstração nas aulas de matemática do ensino médio e também como a aplicação de um teorema relativamente simples pode simplificar a solução de um problema mais elaborado.
\end{abstract}

Palavras-chave: O Teorema de Stewart, O Teorema de Heron, Cevianas, Arbelos, O problema das circunferências tangentes.

\section{Introdução}

Os alunos do ensino médio brasileiro geralmente não conhecem teoremas, não sabem demonstrar e têm dificuldades para solucionar problemas aplicados utilizando conhecimentos matemáticos previamente assimilados. Para exemplificar, cita-se o trabalho de Oliveira [5], que aplicou uma atividade centrada no Teorema de Stewart a alunos do segundo ano do ensino médio público, e constatou que esses estudantes, mesmo já tendo estudado trigonometria em triângulos quaisquer, não foram capazes de empregar a Lei dos Cossenos para solucionar os problemas propostos.

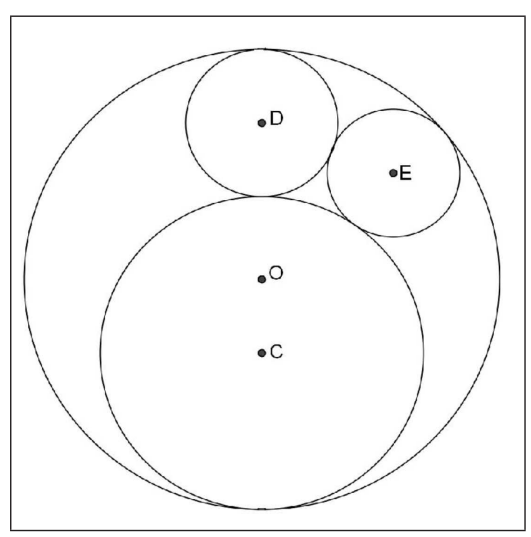

Figura 1: O problema das circunferências tangentes.
Um dos problemas dessa atividade é o problema das quatro circunferências tangentes, enunciado a seguir.

O problema das circunferências tangentes: Calcular o raio da circunferência de centro $E$, sabendose que o raio da circunferência de centro $D$ mede $1 \mathrm{~cm}$ e o raio da circunferência de centro $C$ mede $2 \mathrm{~cm}$. As três circunferências são tangentes entre si e tangentes à circunferência de centro $O$, como ilustra a Figura 1 [3].

Apresenta-se nas próximas seções o Teorema de Stewart, sua demonstração e utiliza-se esse teorema para demonstrar o Teorema de Heron e para solucionar problemas aplicados, como o problema das circunferências tangentes.

\footnotetext{
*Parte desta pesquisa foi financiada pela CAPES
} 


\section{O Teorema de Stewart}

Teorema 2.1 (Stewart) Dados um triângulo $A B C$ e um ponto $D$ do lado $A B$, vale a relação

$$
a^{2} n+b^{2} m-d^{2} c=c m n,
$$

onde $a, b$ e c são as medidas dos lados, d é a ceviana $C D$ e $m$ e $n$ são os segmentos determinados pela ceviana $C D$ no lado $A B$ [3][4][6].

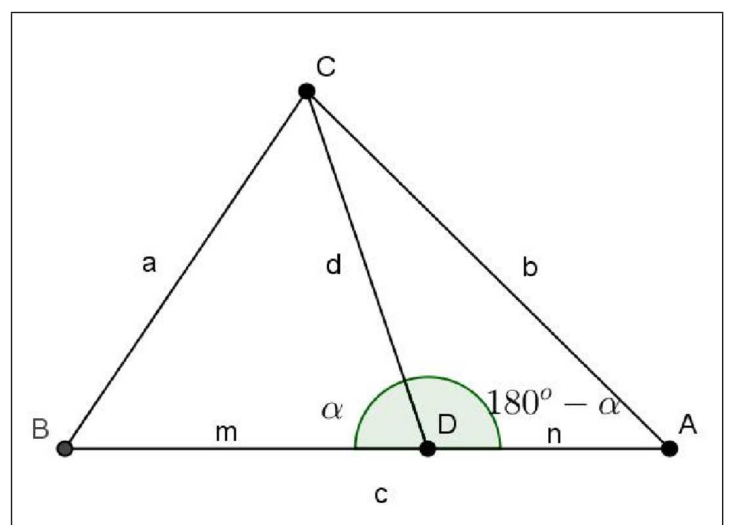

Figura 2: Demonstração do Teorema de Stewart pela Lei dos Cossenos.

\section{$\underline{\text { Demonstração }}$}

Na Figura 2, $m$ e $n$ são as medidas dos segmentos determinados pela ceviana $d$ no lado $A B, \alpha$ é a medida do ângulo $B D C$ e $180^{\circ}-\alpha$ é a medida do ângulo $A D C$. Aplicando-se a Lei dos Cossenos aos triângulos $B D C$ e $A D C$, obtém-se, respectivamente,

$$
\begin{gathered}
a^{2}=m^{2}+d^{2}-2 m d \cos (\alpha) \\
b^{2}=n^{2}+d^{2}-2 n d \cos \left(180^{0}-\alpha\right) .
\end{gathered}
$$

Sabendo-se que $\cos (\alpha)=-\cos \left(180^{\circ}-\alpha\right)$, pode-se reescrever a Equação (2) como

Subtraindo-se as Equações (1) e (3), chega-se a

$$
\begin{aligned}
a^{2}-b^{2} & =m^{2}-n^{2}-2 m d \cos (\alpha)-2 n d \cos (\alpha), \\
\cos (\alpha) & =\frac{b^{2}+m^{2}-a^{2}-n^{2}}{2 d(m+n)} .
\end{aligned}
$$

Substituindo-se a Equação (4) na Equação (1), tem-se que:

$$
\begin{aligned}
a^{2} & =m^{2}+d^{2}-2 m d \frac{b^{2}+m^{2}-a^{2}-n^{2}}{2 d(m+n)} ; \\
a^{2}(m+n) & =m^{2}(m+n)+d^{2}(m+n)-b^{2} m-m^{3}+a^{2} m+n^{2} m ; \\
a^{2} m+a^{2} n & =m^{3}+m^{2} n+d^{2} m+d^{2} n-b^{2} m-m^{3}+a^{2} m+n^{2} m ; \\
a^{2} n+b^{2} m & =d^{2}(m+n)+m n(m+n) .
\end{aligned}
$$

Como $m+n=c$, reescreve-se a Equação (5) como

$$
a^{2} n+b^{2} m-d^{2} c=c m n \text {. }
$$

\section{O Teorema de Heron}

Teorema 3.1 (Heron) A área $S$ de um triângulo $A B C$ qualquer é dada por

$$
S_{A B C}=\sqrt{p(p-a)(p-b)(p-c)},
$$

sendo $p=\frac{a+b+c}{2}$ o semiperímetro do triângulo e $a, b$ e $c$ as medidas dos lados. 


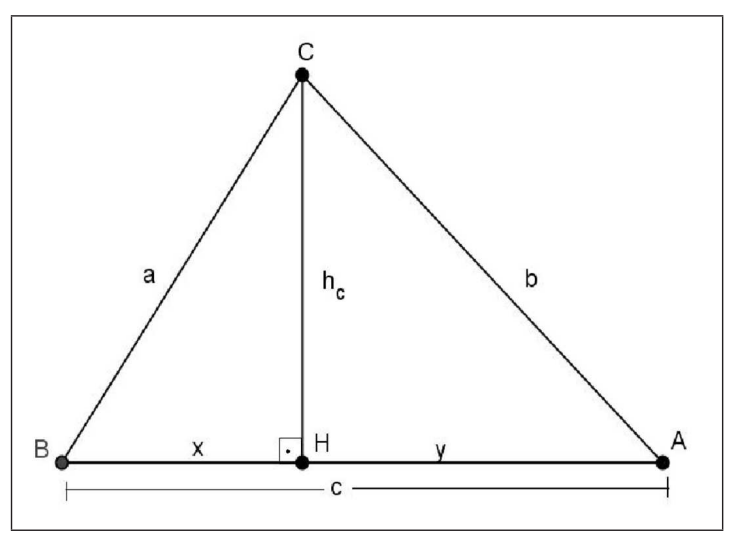

Figura 3: Demonstração do Teorema de Heron.

\section{Demonstração}

Seja o triângulo $A B C$ de base $c$ e altura $h_{c}$, ilustrado na Figura 3. Aplicando-se o Teorema de Pitágoras aos triângulos $B H C$ e $A H C$, obtém-se, respectivamente,

$$
a^{2}=x^{2}+h_{c}^{2}
$$

e

$$
b^{2}=y^{2}+h_{c}^{2} .
$$

Subtraindo-se as Equações (7) e (8), temse que

$$
a^{2}-b^{2}=x^{2}-y^{2}=(x+y)(x-y) .
$$

Como $x+y=c$, então

$$
y=c-x .
$$

Substituindo-se a Equação (10) na Equação (9), obtém-se

$$
x=\frac{a^{2}-b^{2}+c^{2}}{2 c} \text {. }
$$

Substituindo-se a Equação (11) na Equação (10), tem-se que

$$
y=\frac{-a^{2}+b^{2}+c^{2}}{2 c} .
$$

Aplicando-se o Teorema de Stewart ao triângulo $A B C$, obtém-se

$$
a^{2} y+b^{2} x-h_{c}^{2} c=c x y .
$$

Substituindo-se as Equações (11) e (12) na Equação (13), tem-se que:

$$
\begin{aligned}
a^{2} \frac{-a^{2}+b^{2}+c^{2}}{2 c}+b^{2} \frac{a^{2}-b^{2}+c^{2}}{2 c}-h_{c}^{2} c & =c \frac{a^{2}-b^{2}+c^{2}}{2 c} \frac{-a^{2}+b^{2}+c^{2}}{2 c} \\
-2 a^{4}+4 a^{2} b^{2}+2 a^{2} c^{2}-2 b^{4}+2 b^{2} c^{2}-4 h_{c}^{2} c^{2} & =-a^{4}-b^{4}+c^{4}+2 a^{2} b^{2} ; \\
4 h_{c}^{2} c^{2} & =-a^{4}-b^{4}-c^{4}+2 a^{2} b^{2}+2 a^{2} c^{2}+2 b^{2} c^{2} .
\end{aligned}
$$

Somando-se e subtraindo-se $2 a^{2} c^{2}$ ao lado direito da Equação (14), obtém-se:

$$
\begin{aligned}
& 4 h_{c}^{2} c^{2}=4 a^{2} c^{2}-\left(a^{4}+b^{4}+c^{4}-2 a^{2} b^{2}+2 a^{2} c^{2}-2 b^{2} c^{2}\right) ; \\
& 4 h_{c}^{2} c^{2}=4 a^{2} c^{2}-\left[\left(a^{4}+2 a^{2} c^{2}+c^{4}\right)-2 b^{2}\left(a^{2}+c^{2}\right)+b^{4}\right] ; \\
& 4 h_{c}^{2} c^{2}=4 a^{2} c^{2}-\left[\left(a^{2}+c^{2}\right)^{2}-2\left(a^{2}+c^{2}\right) b^{2}+b^{4}\right] ; \\
& 4 h_{c}^{2} c^{2}=4 a^{2} c^{2}-\left(a^{2}+c^{2}-b^{2}\right)^{2} ; \\
& 4 h_{c}^{2} c^{2}=\left[2 a c+\left(a^{2}+c^{2}-b^{2}\right)\right]\left[2 a c-\left(a^{2}+c^{2}-b^{2}\right)\right] ; \\
& 4 h_{c}^{2} c^{2}=\left[\left(a^{2}+2 a c+c^{2}\right)-b^{2}\right]\left[-\left(a^{2}-2 a c+c^{2}\right)+b^{2}\right] ; \\
& 4 h_{c}^{2} c^{2}=\left[(a+c)^{2}-b^{2}\right]\left[b^{2}-(a-c)^{2}\right] ; \\
& 4 h_{c}^{2} c^{2}=(a+c+b)(a+c-b)(b+a-c)(b-a+c) .
\end{aligned}
$$


O perímetro do triângulo $A B C$ é dado por $2 p=a+b+c$. Logo:

$$
\begin{aligned}
& a+c-b=a+b+c-2 b=2 p-2 b=2(p-b) \\
& b+a-c=a+b+c-2 c=2 p-2 c=2(p-c) \\
& b-a+c=a+b+c-2 a=2 p-2 a=2(p-a)
\end{aligned}
$$

Substituindo-se as igualdades (16), (17) e (18) na Equação (15), tem-se que:

$$
\begin{aligned}
4 h_{c}^{2} c^{2} & =2 p 2(p-b) 2(p-c) 2(p-a) ; \\
h_{c}^{2} & =\frac{4}{c^{2}} p(p-a)(p-b)(p-c) ; \\
h_{c} & =\frac{2}{c} \sqrt{p(p-a)(p-b)(p-c)} .
\end{aligned}
$$

Como a área $S$ do triângulo $A B C$ pode ser calculada pelo semiproduto de um lado pela altura relativa a esse lado, tem-se que

$$
S_{A B C}=\frac{1}{2} h_{c} c .
$$

Substituindo-se a Equação (19) em (20), obtém-se:

$$
\begin{array}{r}
S_{A B C}=\frac{1}{2} c \frac{2}{c} \sqrt{p(p-a)(p-b)(p-c)} ; \\
S_{A B C}=\sqrt{p(p-a)(p-b)(p-c)} .
\end{array}
$$

\section{Aplicações}

\subsection{O problema das circunferências tangentes}

Sejam $x$ a medida do raio da circunferência de centro $E$ ilustrada na Figura $4, D \hat{O} E=\alpha$ e $C \hat{O} E=\beta$. Verifica-se facilmente que o raio da circunferência de centro $O$ mede $3 \mathrm{~cm}$ e que:

$O E=O T-E T=3-x=z ; \quad D E=1+x=a ; C E=2+x=b ; \quad O D=2=m ; \quad O C=1=n$.

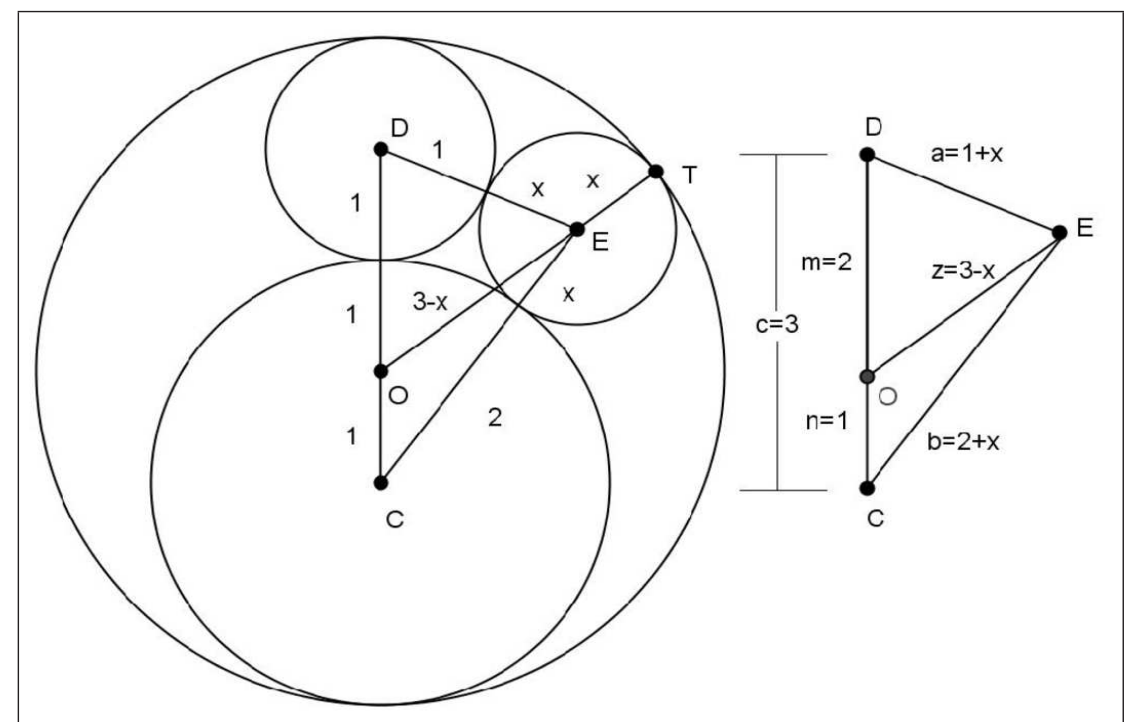

Figura 4: Triângulo $C D E$ cujos vértices são os centros de três das circunferências tangentes. 
Aplicando-se o Teorema de Stewart ao triângulo $C D E$ da Figura 4, obtém-se:

$$
\begin{aligned}
a^{2} n+b^{2} m-z^{2} c & =c m n ; \\
(1+x)^{2}(1)+(2+x)^{2}(2)-(3-x)^{2}(3) & =3(2)(1) ; \\
1+2 x+x^{2}+8+8 x+2 x^{2}-27+18 x-3 x^{2} & =6 ; \\
28 x & =24 ; \\
x & =\frac{6}{7} .
\end{aligned}
$$

\subsection{Demonstração de outros teoremas}

Emprega-se agora o Teorema de Stewart para demonstrar o Teorema 4.1, proposto em [6].

Teorema 4.1 A soma dos quadrados das medidas dos lados de um paralelogramo é igual à soma dos quadrados das medidas das diagonais.

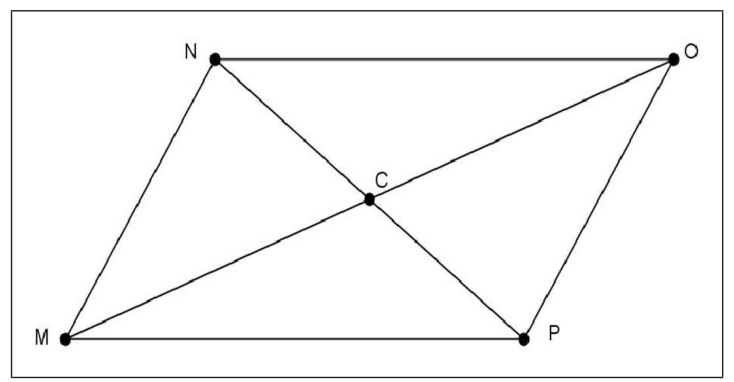

Figura 5: Paralelogramo MNOP e suas diagonais $M O$ e $N P$.

$$
\begin{aligned}
a^{2} \frac{d_{1}}{2}+b^{2} \frac{d_{1}}{2}-\left(\frac{d_{2}}{2}\right)^{2} d_{1} & =d_{1} \frac{d_{1}}{2} \frac{d_{1}}{2}, \\
\frac{a^{2}}{2}+\frac{b^{2}}{2}-\frac{d_{2}^{2}}{4} & =\frac{d_{1}^{2}}{4}
\end{aligned}
$$

e

$$
\begin{aligned}
a^{2} \frac{d_{2}}{2}+b^{2} \frac{d_{2}}{2}-\left(\frac{d_{1}}{2}\right)^{2} d_{2} & =d_{2} \frac{d_{2}}{2} \frac{d_{2}}{2}, \\
\frac{a^{2}}{2}+\frac{b^{2}}{2}-\frac{d_{1}^{2}}{4} & =\frac{d_{2}^{2}}{4} .
\end{aligned}
$$

Somando-se as Equações (22) e (23), obtém-se:

$$
\begin{aligned}
2 \frac{a^{2}}{2}+2 \frac{b^{2}}{2}-\frac{d_{1}^{2}}{4}-\frac{d_{2}^{2}}{4} & =\frac{d_{1}^{2}}{4}+\frac{d_{2}^{2}}{4} ; \\
2\left(a^{2}+b^{2}\right) & =d_{1}^{2}+d_{2}^{2} .
\end{aligned}
$$

\subsection{Arbelos}

Arbelos, do grego "faca de sapateiro" - Figura 6 (a), é uma região plana delimitada por três semicircunferências, como ilustra a Figura 6 (b). Acredita-se que Arquimedes tenha sido o primeiro a estudar suas propriedades matemáticas.

Pode-se utilizar o Teorema de Stewart para relacionar os raios $r_{1}, r_{2}$ e $r_{1}+r_{2}$ de uma arbelos com o raio $r$ de uma circunferência inscrita [1], como ilustra a Figura 7. Constatase que o triângulo cujos vértices são os centros das semicircunferências de raios $r_{1}$ e $r_{2}$ e da 


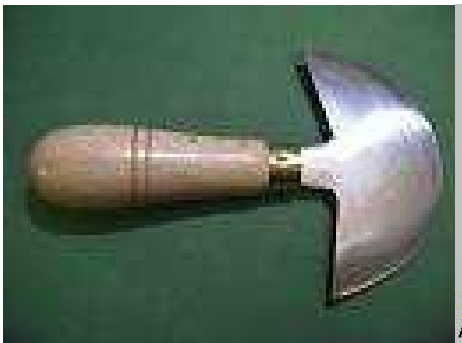

(a)

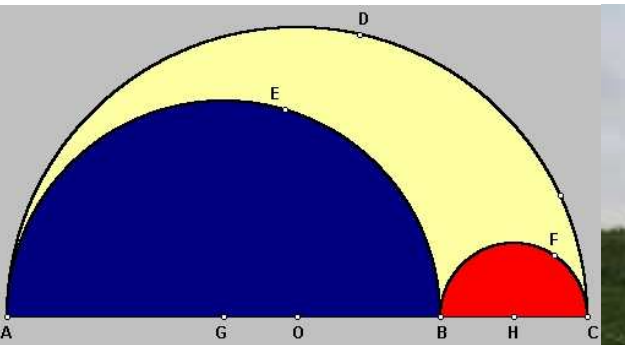

(b)

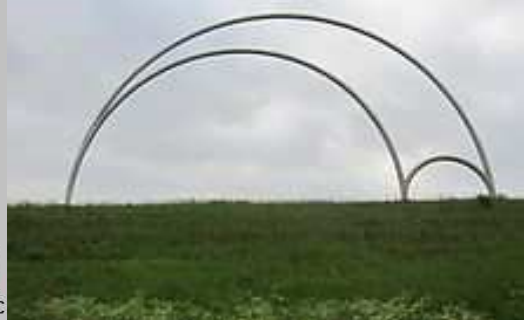

(c)

Figura 6: (a) Faca de sapateiro [2], (b) arbelos [7] e (c) escultura na Holanda [8].

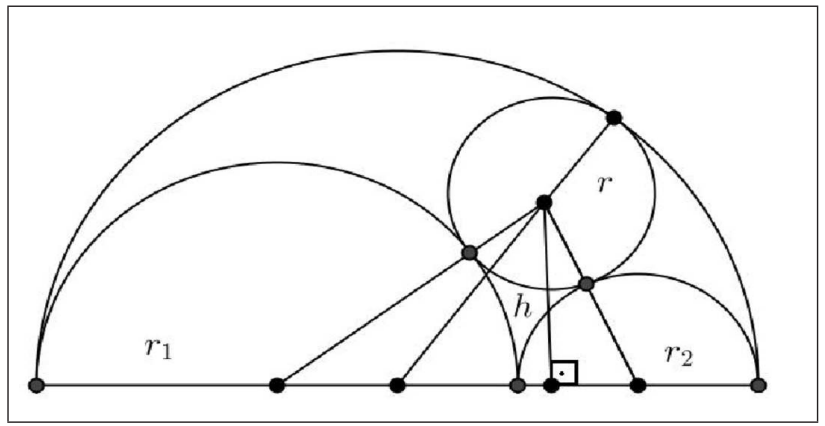

Figura 7: Circunferência inscrita em uma arbelos.

circunferência de raio $r$, tem lados de medidas $r+r_{1}, r+r_{2}$ e $r_{1}+r_{2}$. Aplicando-de o Teorema de Stewart a esse triângulo considerando-se a ceviana de medida $r_{1}+r_{2}-r$, tem-se que

$$
\left(r+r_{1}\right)^{2}\left(r_{1}\right)+\left(r+r_{2}\right)^{2}\left(r_{2}\right)-\left(r_{1}+r_{2}-r\right)^{2}\left(r_{1}+r_{2}\right)=r_{1} r_{2}\left(r_{1}+r_{2}\right) .
$$

Desenvolvendo-se algebricamente a igualdade (25), obtém-se:

$$
\begin{aligned}
4 r r_{1} r_{2}+4 r r_{1}^{2}+4 r r_{2}^{2}-3 r_{1}^{2} r_{2}-3 r_{1} r_{2}^{2} & =r_{1} r_{2}\left(r_{1}+r_{2}\right) ; \\
4 r\left(r_{1} r_{2}+r_{1}^{2}+r_{2}^{2}\right)-3 r_{1} r_{2}\left(r_{1}+r_{2}\right) & =r_{1} r_{2}\left(r_{1}+r_{2}\right) ; \\
4 r\left(r_{1} r_{2}+r_{1}^{2}+r_{2}^{2}\right) & =4 r_{1} r_{2}\left(r_{1}+r_{2}\right) ; \\
r & =\frac{r_{1} r_{2}\left(r_{1}+r_{2}\right)}{r_{1}^{2}+r_{2}^{2}+r_{1} r_{2}} .
\end{aligned}
$$

Utilizando-se o Teorema de Heron, pode-se mostrar agora que, na Figura 7, $h=2 r$.

A área $S$ do triângulo cujos vértices são os centros das circunferências de raios $r_{1}, r_{2}$ e $r$ é dada por

$$
S_{\Delta}=\frac{1}{2} h\left(r_{1}+r_{2}\right) .
$$

Aplicando-se o Teorema de Heron a esse triângulo, tem-se que

$$
S_{\Delta}=\sqrt{\left(r+r_{1}+r_{2}\right) r r_{1} r_{2}} .
$$


Substituindo-se a Equação (26) em (28), obtém-se:

$$
\begin{aligned}
& S_{\Delta}=\sqrt{\left(\frac{r_{1} r_{2}\left(r_{1}+r_{2}\right)}{r_{1}^{2}+r_{2}^{2}+r_{1} r_{2}}+r_{1}+r_{2}\right) \frac{r_{1} r_{2}\left(r_{1}+r_{2}\right)}{r_{1}^{2}+r_{2}^{2}+r_{1} r_{2}} r_{1} r_{2}} ; \\
& S_{\Delta}=\sqrt{\frac{\left(r_{1}+r_{2}\right)\left(r_{1}^{2}+2 r_{1} r_{2}+r_{2}^{2}\right)}{r_{1}^{2}+r_{2}^{2}+r_{1} r_{2}} \frac{r_{1} r_{2}\left(r_{1}+r_{2}\right)}{r_{1}^{2}+r_{2}^{2}+r_{1} r_{2}} r_{1} r_{2} ;} \\
& S_{\Delta}=\sqrt{\frac{\left(r_{1}+r_{2}\right)\left(r_{1}+r_{2}\right)^{2}}{r_{1}^{2}+r_{2}^{2}+r_{1} r_{2}} \frac{r_{1} r_{2}\left(r_{1}+r_{2}\right)}{r_{1}^{2}+r_{2}^{2}+r_{1} r_{2}} r_{1} r_{2} ;} \\
& S_{\Delta}=\frac{\left(r_{1}+r_{2}\right)^{2} r_{1} r_{2}}{r_{1}^{2}+r_{2}^{2}+r_{1} r_{2}} .
\end{aligned}
$$

Igualando-se as Equações (27) e (29) e empregando-se a Equação (26), conclui-se que:

$$
\begin{aligned}
& h=2 \frac{\left(r_{1}+r_{2}\right) r_{1} r_{2}}{r_{1}^{2}+r_{2}^{2}+r_{1} r_{2}} \\
& h=2 r .
\end{aligned}
$$

\section{Conclusão}

A solução do problema das quatro circunferências tangentes, assim como a relação entre os raios das semicircunferências que definem uma arbelos com o raio da circunferência inscrita, é simplificada com o uso do Teorema de Stewart, o qual também permite demonstrar outros teoremas, como o Teorema de Heron. Ressaltou-se neste artigo a demonstração de teoremas com o intuito de incentivar os professores de matemática do ensino médio a incorporar efetivamente a demonstração no processo ensino-aprendizagem e também a usar os teoremas demonstrados na solução de problemas aplicados.

\section{Referências}

[1] A Matemática Pura, "Brahmagupta, Heron e alguma aplicações interessantes", 2014. Disponível em: http://amatematicapura.blogspot.com.br/2012/07/brahmagupta-heron-ealgumas-aplicacoes.html. Acesso em: 19 de fevereiro de 2014.

[2] Bowstock, "Round knife", 2014. Disponível em: http://www.bowstock.co.uk/acatalog/Knives.html. Acesso em: 19 de fevereiro de 2014.

[3] O. Dolce; J.N. Pompeo, "Fundamentos de Matemática Elementar", v. 9, $6^{a}$ ed, Atual, São Paulo, 2005.

[4] A.C. Morgado; E. Wagner; M. Jorge, "Geometria II", 4ª ed, VestSeller, São Paulo, 2009.

[5] C.A.M. de Oliveira, "Os teoremas de Stewart e de Heron e o cálculo da área de um triângulo em função dos lados", Dissertação de Mestrado, PROFMAT-UTFPR-CT, 2014.

[6] A.S. Posamentier; C.T. Salkind, "Challenging problems in geometry", Dover, New York, 1996.

[7] The University of Georgia, "Arbelos: the shoemaker's knife", 2014. Disponível em: http://jwilson.coe.uga.edu/EMT668/EMAT6680.2000/Westmoreland/Essay1/Essay1.html. Acesso em: 19 de fevereiro de 2014.

[8] Wikipedia, "Arbelos", 2014. Disponível em: http://en.wikipedia.org/wiki/Arbelos. Acesso em: 19 de fevereiro de 2014. 